\title{
Living Versus Inanimate: The Information Border
}

\author{
Gérard Battail
}

Received: 18 May 2009 / Accepted: 10 June 2009 /

Published online: 3 October 2009

(C) Springer Science + Business Media B.V. 2009

\begin{abstract}
The traditional divide between nature and culture restricts to the latter the use of information. Biosemiotics claims instead that the divide between nature and culture is a mere subdivision within the living world but that semiosis is the specific feature which distinguishes the living from the inanimate. The present paper is intended to reformulate this basic tenet in information-theoretic terms, to support it using information-theoretic arguments, and to show that its consequences match reality. It first proposes a 'receiver-oriented' interpretation of semiosis. This interpretation implies that the means for recording, storing and processing information exclusively reside in the living world (extended so as to include the artefacts it produces). Then it may be argued that the main difference between the inanimate world and the living one lies in the fact that the very existence of the latter relies on information, which on the contrary is not relevant to the former. Thus, besides matter and energy, information is an entity irreducible to them which must be taken into account in any attempt for describing and understanding life. Information can interact with the real world only provided it is borne by some physical support: it must be 'physically inscribed'. Contrary to matter and energy, information can be shared, not necessarily exchanged, so a same information can be borne by a number of distinct supports. Any living thing possesses means for recording, storing and processing information which are necessary for keeping it alive and securing its progeny. In particular, its genome contains hereditary information, can be replicated, and instructs the construction and maintenance of a phenotype. The simultaneous existence of a phenotype and of a genome, where the latter bears the symbolic description of the former,
\end{abstract}

This paper was presented at the 9-th Biosemiotics Gathering in Prague, June 30-July 4, 2009.

G. Battail $(\varangle)$

E.N.S.T., Paris, France

e-mail: gbattail@club-internet.fr 
is mandatory for enabling the self-reproduction of an organism. Bearing and using information then endows a living thing with the ability to decrease the physical entropy, hence to act as Maxwell's demon. Not only its own life is maintained against physical entropy, but its self-reproduction multiplies clones of the demon. Taking information as the entity which differentiates the living from the inanimate also supports Rovelli's 'relational' interpretation of quantum physics. Experimental apparatuses then appear as informationtheoretic channels from the inanimate world to a living observer. Besides having its own perspective (as stated by Rovelli), each of these channels has its own horizon because its capacity is necessarily finite. As another consequence, we may assert that the physicists' quest of a 'theory of everything' is doomed to failure since, for lack of considering information as a relevant entity, physicists deny the living world, hence themselves.

Keywords Living vs inanimate divide - Receiver-oriented interpretation of semiosis • Information theory • Symbolic information • Maxwell's demon

\section{Introduction}

This paper is intended to revisit and support by information-theoretic arguments the basic tenet of biosemiotics that semiosis is the specific feature which differentiates the living from the inanimate. Some of its consequences as regards the relationship of biology and physics are also examined.

Can we delineate within the physical world a border between the inanimate and living worlds? The living world belongs to the physical world in which it is embedded, but biosemiotics states that it is radically distinct from the inanimate world as the unique place where semiosis takes place. We propose a 'receiver-oriented' interpretation of semiosis. It entails that the living world is unique as made of objects which exist only insofar as they can store, process and communicate information. Understanding the living world thus needs considering information as its distinctive entity; on the contrary, the inanimate world ignores it. At variance with the current, rather fuzzy, criteria for characterizing life (see, for instance, how difficult it is to conceive criteria for detecting extraterrestrial life (Morange 2007)), this remark provides means for sharply distinguishing the living from the inanimate.

In the whole paper, we assume that semiosis is the feature which uniquely distinguishes the living from the inanimate, understanding semiosis according to our 'receiver-oriented' interpretation. Then information theory, which deals with information as a mathematical entity and enables measuring it, is fully relevant to the living world. It is the main framework of this study. It should be taken in a broad sense as including, besides a formal content which can been found in the seminal work of Shannon (1948) or in textbooks, e.g., (Cover and Thomas 1991), the lessons that communication engineers learned from their own practice. Accordingly, we think of information as the entity which links abstraction with the physical world. No information exists unless it is inscribed 
on some physical support. Information cannot be used or processed in any way outside physical devices: any operation which involves information needs be physically implemented. A less important lesson from engineering practice is that solving a difficult problem needs complex implementation: the more difficult is the former, the more complex the latter.

Neurologists, interestingly, reached the same conclusions as engineers. $\mathrm{Hu}-$ mans have long thought of ideas as devoid of any physical support, having an existence of their own. We now understand that this is an illusion. The human brain is an object of huge complexity and its operation entirely escapes our consciousness, but the human thought is indeed physically inscribed in neurons. Idealism is no longer a tenable position. The Platonic world of Ideas is not actually separated from the physical world: information is the entity which bridges them. More precisely, information is the means by which ideas can reside in the physical world and interact with it.

Information will be considered throughout this paper as providing the link between physical-chemical processes and those involving meaning. At variance with Peircean semiotics, information theory is a quantitative and mathematical science which moreover has some relationship with physics, especially through the concept of entropy. This reason and its extraordinary fruitfulness in communication engineering should prompt biologists to accept it. Unfortunately, engineering is terra incognita for many of them although, rather obviously, life is an outstanding expert in solving engineering problems.

\section{A Receiver-Oriented Interpretation of Semiosis}

We suggest, starting from the definition of semiosis as the production of signs (Barbieri 2008a), to restrict signs to events which can be received, i.e., which can change in any way the state of some device or being, referred to as a receiver, thereby delivering information to it. This interpretation follows from remarks we already formulated (Battail 2008b). It implies that a sign does not exist independently of a receiver. The statement that semiosis is the basic feature which distinguishes the living from the inanimate then implies that

information-receiving devices exclusively exist inside the living world.

Assuming that the information provided by a sign controls the receiver's state implies that it is recorded, at least temporarily. Then a receiver includes a memory in the engineering meaning of the word, ${ }^{1}$ so receiver-oriented semiosis means production of signs which can result in information being recorded by adequate receivers. Moreover, the content of the receiver memory must interact with something outside it: recording is not an end in itself. In other words, it should in turn be used in order to produce signs.

To make the above statement valid needs extending the definition of the living world to include, besides living things, the artefacts they may produce.

\footnotetext{
${ }^{1}$ A device which can store information, not its content.
} 
Human-made devices and systems are especially considered as parts of the extended living world insofar as they record, store and process information. This extension is similar to that proposed by Dawkins who, for instance, includes in the beaver's phenotype the dams it builds (Dawkins 1982). Of course, these artefacts are included within the living world only as tools or prostheses associated with true living beings.

Mere recording does not suffice for receiver-oriented semiosis to take place. Let us consider tracks, prints, sediments, fossils, ..., which result from purely physical-chemical processes and provide information about the occurrence of past events or, similarly, the electromagnetic radiation emitted by a star which indicates its chemical composition and its speed relatively to the observer. In any case, these are raw data to be further processed for making the information they contain available. We may think of such data as potential information. We shall make later more precise what kind of processing can turn potential into usable information. The reinterpretation of the concept of semiosis we propose is intended to shift the emphasis towards the receiving end: the produced signs need be perceived. It complies with Shannon's statement that 'the fundamental problem of communication is that of reproducing at one point [...] a message selected at another point' (my italics) (Shannon 1948). Reception is actually the crucial step in a communication since the choice of a sign which has been made at the transmitting end must be inferred by the receiver. Inferring what sign has been produced implies knowing what signs can occur and dealing with them as chance events. Moreover, the receiver does not escape outer influences so the signs which are produced, far from being perceived in isolation, are received in the presence of external perturbing factors, which collectively result in a spurious signal referred to as noise. Noise can only be dealt with as random so the receiving process is basically probabilistic. It has thus a nonzero probability of failure, and the recorded result has a nonzero probability of error, i.e., of being different from the sign actually produced. The physical-chemical processes which produce tracks, prints, sediments, fossils, or radiations ..., are of an entirely different kind since no receiving processes in the meaning just defined take place prior to recording. They will result in usable information only when they interact with outer objects, i.e., when they are received by predators, hunters, detectives, geologists or astrophysicists, who all are living beings.

Notice that reception is by no means a passive function, as it may seem at first sight. On the contrary, it implies a process leading to a decision. The difficulty of the reception processes entails that receivers are intrinsically complex. Restricting the possible receivers of information to the living world (properly extended) entails that it exclusively gathers complex objects, as the living world actually does.

We started from a minimal definition of semiosis: the production of signs. Biosemioticians consider meaning as the main attribute of signs and thus think of it as the very essence of semiosis. We content ourselves here with literal communication: the receivers we consider have as mere task to identify signs, not to interpret them. This restriction enables using information theory, which 
ignores semantics. Making signs available to their destination is a necessary and nontrivial step in a communication process although not the final one, which involves semantics. Information thus appears as a necessary intermediate in semantic communication. The semantic content associated with an informational support is arbitrary but cannot be communicated independently of its support. This point of view has been expounded at greater length in (Battail 2008b).

'Semiosis' basically means that some object, referred to as a sign, is not considered for what it is (e.g., its physical-chemical composition), but stands for something else according to a convention. The sign which represents an object is arbitrary. This object has no relationship with the sign except the one that the convention states, and it may be abstract as well as concrete. The point of view of information theory consists of ignoring semantics, thus leaving the represented object indeterminate. The sign is assumed to belong to some set of possible signs and only their number is considered as relevant. For instance, a binary symbol expresses a choice between two options, hence a sequence of $k$ binary symbols corresponds to $2^{k}$ different options at most. Biosemiosis in Barbieri's meaning implies the existence of natural conventions, a seeming oxymoron. This existence has far-reaching consequences, tending to blur the frontier between culture and nature, and suggesting that the truly significant divide lies between the living and the inanimate (Barbieri 2008b, c).

\section{The Living versus Inanimate Divide}

We propose Fig. 1 as a picture of the physical world. It is divided into the inanimate and living worlds. The basic tenet of biosemiotics together with the reinterpretation of semiosis just proposed entails that information-receiving devices and systems are exclusively located in the (extended) living world. Three basic entities are then relevant: matter, energy and information. ${ }^{2}$

Matter and energy are conserved, so matter and energy in the living world have been taken out from the inanimate world, or vice-versa. For instance, plants use the solar energy to synthesize organic molecules from carbon dioxide and water. These are taken out from the inanimate world. The synthesized organic matter makes up tissues and maintains life processes of the plant thanks to the chemical energy stored in molecules like sugars. When the plant life ends, for instance if it burns, its constituents return to the inanimate world. It is why a double arrow has been drawn in Fig. 1 for expressing that matter and energy can flow from the inanimate world to the living one, or vice-versa.

As regards information, a single arrow has been drawn in Fig. 1 from the inanimate world to the living one in order to express that the flow of

\footnotetext{
${ }^{2}$ As far as we know, that information should be considered, besides matter and energy, as a fundamental entity for describing the physical world has first been proposed by Kenneth Ewart Boulding (1956).
} 
Fig. 1 Splitting the physical world into inanimate and living worlds. $M, E$ and $I$ stand for matter, energy and information, respectively
Physical world

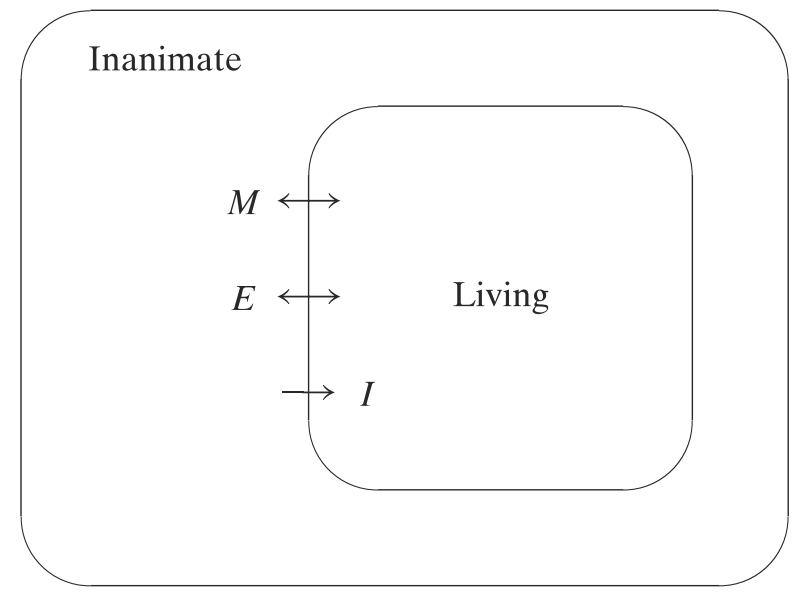

information is unidirectional. We assumed indeed that information receivers necessarily belong to the living world hence that no information can reside in the inanimate world: only information-generating events, referred to above as potential information, occur in it. Would information be conserved, the quantity of information present in the living world would increase indefinitely. But it is not so. New information can be written on a support so as to replace the information already stored in it, which is then lost. At variance with matter and energy, information can thus be annihilated. Such an event may occur in the course of information processing, but also spontaneously because there is a nonzero probability that noise writes spurious information on any support, progressively eliminating its previous content (this results, for instance, in the exponential decrease of DNA capacity, which entails the need for genomic error-correcting codes; see Battail 2007, 2008a, c). Another distinctive feature of information is that it can be shared, i.e., an information written on some support can be copied on another one while still remaining present on the initial support. Then the same information can be borne by several supports, a fundamental property which radically differentiates information from matter and energy which, being conserved, can only be exchanged. In other words, information can proliferate.

This vision of the divide between living and inanimate is in good agreement with sensible intuition. The inanimate world appears indeed as blind, devoid of means to receive information, while information is ubiquitous in the living world. Another big difference is that the objects of the living world are organized, at variance with those of the inanimate world, but we shall see in "What Is Life?", below, that this fundamental property, too, is a consequence of the ability of objects of the living world to store and process information. 


\section{Information Is Vital}

The biological functions which subtend life at the molecular level, namely, transcription of DNA into RNA and translation of messenger RNA into a polypeptidic chain (later becoming a protein), involve information-bearing objects and need information. Not only DNA and RNA are molecular memories which contain information, but the agents which implement these functions, the enzymes, need information for performing them. These miracle-makers of molecular biology act indeed as receivers in the information-theoretic meaning as being able to recognize specific molecular structures with extreme accuracy. Much of the biological literature seems to be content with naming enzymes in terms of the function they perform. To name is not to explain, however, and engineers experienced how difficult are recognition tasks, as recalled in (Battail 2008b). How enzymes work is far from trivial and the detailed understanding of the way they recognize molecular structures would be of great interest. Information is clearly at the heart of this enzymatic function.

We may think of recognition as extending the function of reception to objects of dimension higher than 1 . In a unidimensional space, reception involves the difficulty due to the necessity of inferring, in the presence of noise, what message has been sent. Besides this difficulty, reception becomes a much more complicated task in physical spaces of higher dimension. In 2-dimensional space, objects need often be recognized up to a change of orientation; they may also incur a change in scale (dilatation or contraction) or more or less important differences in shape (think, for instance, of the many different fonts that can be used to represent the letters of the alphabet). Still further difficulties arise in 3-dimensional space. Considering for instance visual recognition, not only objects can be seen at a variable distance (resulting in a scale difference) and with an arbitrary orientation, but under different angles and perspectives. A same 3-dimensional object is thus seen as an infinity of possible 2-dimensional patterns, and yet should be identified. Moreover, objects mutually hide each other (see René Magritte's canvas La Grande Guerre) so their identification should often be performed from the vision of only a part of them. Yet, recognition of 3-dimensional objects is of vital importance in at least three instances: for enzymes to recognize molecular structures, for the immunity system to detect pathogens or potentially dangerous intruders, and for individual living beings to sense their environment.

Indeed, the operation of living things deeply relies on recognition abilities. Keeping an organism alive clearly involves information of various kinds about its physical and biological environment, as well as about its internal state, used in many feedback loops. For instance, an animal must perceive physical parameters like gravity, temperature, pressure, ..., but should also be able to recognize individuals of its own species, detect possible predators, preys or sexual mates so as to behave accordingly. In all such instances, information is necessary for the survival of an individual and its progeny. The ability of recognition is also of crucial importance for immunity since reliably recognizing 
the self from the non-self is a necessary condition for defending an organism against pathogens.

Maintaining an individual's life thus heavily depends on its recognition ability, hence on its information-theoretic performance. The complexity of the means which need be implemented for reliably performing recognition tasks is greatly overlooked by biologists. Books report apparently miraculous abilities of living beings in recognition tasks. For instance, those which deal with kin selection consider as a matter of fact that animals, or even less evolved living things, are able to recognize kinship. None of them, however, investigates the means actually implemented to perform this most difficult task.

At the still higher level of ecosystems, information exchanges are of extreme importance, too. The complex relationships which enable diverse elements making together an ecosystem are to a large extent of informational character. Not only information is clearly ubiquitous in the living world, but life would not exist without it, as illustrated by the above examples. In sharp contrast, the interaction between objects of the inanimate world can be fully accounted for by using the physical laws of matter and energy at any scale from the subatomic one to that of the Universe. The physical devices used in communication technology are no exceptions since they are human-made artefacts, hence belong to the extended living world proposed in "A Receiver-Oriented Interpretation of Semiosis".

\section{A Multifaceted Concept}

\section{An Information as an Equivalence Class}

Shannon had an empirical approach to information. He investigated how information can be measured and processed, but did not attempt to define it. He posited at the very beginning of his work that semantics is irrelevant to communication engineering: a communication system intended to make available to its destination a message originating in some source cannot be designed for a specific message. It should instead pre-exist any possible message (Shannon 1948). A messenger has not to know about the meaning of the message he/she bears, just to transport it. This basic postulate has never been questioned in the development of the science of communication engineering rooted in Shannon's work, referred to as information theory. On the contrary, clearly separating information from semantics appears in retrospect as a very fruitful methodological position.

Within this framework, we tried to outline a definition of information which could be of some usefulness in biology (Battail 2008b, c). Basically, we consider information as what is communicated. We first consider a sequence of symbols, i.e., of distinguishable elements taken from a given finite set referred to as the alphabet. Such a sequence is physically inscribed, i.e., each of its symbols is represented by a distinct state of some physical object, device or system. The physical system on which its symbols are inscribed and the size of the alphabet, 
i.e., the number of states this system can assume, are arbitrary. For instance, the sequence of letters

$$
\text { in formation }
$$

uses the 26-letter Latin alphabet and the sequence

$$
110100111011101100110110111111100101101101110000111101001
$$

\section{0}

uses the binary alphabet $\{0,1\}$, the simplest possible one because, needing to be distinct, the symbols of an alphabet must be at least two. In an electrical communication system, an information in binary form would for instance be represented by a sequence of voltages, each associated with a binary symbol $b$, its polarity $s$ depending on it according to $s=(-1)^{b}$; then, a positive (negative) voltage represents the symbol 0 (1).

An inscribed sequence lacks generality as depending on the physical support used in order to represent its symbols and on the alphabet size. As regards the choice of the alphabet, for instance, the above two sequences are actually equivalent since the second one results from converting the letters of the first one into binary according to the 'American Standard Code for Information Interchange' (ASCII) which is currently used in computer memories: every letter of the first sequence has been replaced by a specific 7-binary-digit 'word'.

More important, a given sequence can be transformed into other sequences by encoding processes, in order to endow them with useful properties. The encoding transformations need be reversible, i.e., must enable exactly reverting to the original sequence by properly 'decoding' the encoded sequences. If we consider encoding processes into the same alphabet as the original, the encoded sequences may be shorter than the original (source coding), or longer than it (channel coding). Decreasing the sequence length is useful in order to reduce the size of the memory needed to record it. Increasing its length is but the unavoidable consequence of channel coding, which aims at protecting the original message against errors which possibly affect its symbols.

Given some sequence, infinitely many sequences equivalent to it thus result from its possible encoding, changing the alphabet size and the support on which its symbols are physically inscribed. All the sequences equivalent in this respect to a given sequence constitute an equivalence class, to be referred to as an information. (Considering such a class is a standard means for creating a mathematical object.) Thus defined as an equivalence class, an information is an entity of its own right, regardless of the meaning which may be associated with it, a matter of semantics.

An information is represented by any sequence within its equivalence class. It is suitable to consider as a preferred representative of an information the shortest among all sequences of its equivalence class, written using the simplest possible alphabet, i.e., the binary one. This minimal-length binary sequence will be referred to as the information message associated with the given information. We may consider that its length $k$ quantitatively measures the 
given information, regardless of the length of its original representative. Given a sequence, no explicit means is available in order to determine its information message. Means for obtaining equivalent sequences shorter than the given one exist, but there is no systematic way to make sure that the shortest one has been found. Then only an upper bound on the information quantity a sequence bears is available. An information message is but the binary representation of some natural number, to which each information is uniquely associated. A natural number is a nominal entity in Barbieri's sense (Barbieri 2004, 2008c). Barbieri rightfully notices that the concept of nominal entity is foreign to physics, where numbers generally result from measuring some quantities with a necessarily finite precision. On the contrary, the genome of any living being is represented by an information message, hence by a natural number which is a unique entity.

Considering the information message associated with an information can shed some light on the relation of information with semantics. To this end, we may consider an information message as representing a path within a binary tree. Each of the message symbols, or bit (an acronym for 'binary digit'), can be interpreted as answering a question by 'yes' or 'no'. An information message of length $k$ uniquely represents one among the $2^{k}$ paths of a binary tree, hence specifies one among the $2^{k}$ semantic instances consisting of the answers to $k$ dichotomic questions (think, for example, of identifying a species as a path in its phyletic tree). Although this may seem a rudimentary kind of semantics, $k$ may be very large so an information message of that length specifies one among very many semantic instances. We refer to Battail (2008b) for more details.

\section{Measuring Information}

The length $k$ of the information message associated with an information appears as its natural quantitative measure. This does not suffice to solve the problem of measuring information, however, since it demands that the information message be available. It is so in engineering when channel coding deliberately transforms an information message of length $k$ into a longer word for protecting it against symbol errors, but this case appears as an exception since, in many instances, the information message associated with a given sequence is not explicitly known. Its frequent lack of availability leads to base the measurement of information on other grounds.

Shannon introduced the quantity of information provided by an event as measuring how unexpected this event is. The information quantity $h(x)$ associated with an event $x$ occurring with probability $p$ is defined as

$$
h(x)=\log _{2}(1 / p)=-\log _{2}(p) .
$$

It is expressed in binary units because logarithms to the base 2 are employed in this definition. We name the information unit thus defined shannon (at variance with Shannon who used the acronym bit; we exclusively use 'bit' to designate a binary digit, regardless of the information quantity it may bear). The logarithmic function has been chosen in definition (1) so that, if two inde- 
pendent events $x_{1}$ and $x_{2}$ occur with probabilities $p_{1}$ and $p_{2}$, respectively, the information brought by their joint occurrence, an event having as probability the product $p_{1} p_{2}$ of their individual probabilities, is the sum of the information quantities separately associated with $x_{1}$ and $x_{2}$.

A more important and useful quantity is the mean information quantity associated with a finite random variable $X$, i.e., a variable which possibly assumes $q$ values $x_{i}, i=1,2, \ldots, q$, referred to as its realizations, $x_{i}$ occurring with probability $p_{i}$. Because one of the possible realizations necessarily occurs, the probabilities $\left\{p_{i}\right\}$ sum up to 1 :

$$
\sum_{i=1}^{i=q} p_{i}=1
$$

The mean information quantity associated with $X$ is then the statistical average of the information quantities associated with the realizations, namely,

$$
H(X)=\sum_{i=1}^{i=q} p_{i} h\left(x_{i}\right)=-\sum_{i=1}^{i=q} p_{i} \log _{2}\left(p_{i}\right),
$$

referred to as the entropy ${ }^{3}$ of $X$. Its maximum is achieved when all the events $\left\{x_{i}\right\}$ have the same probability, which equals $1 / q$ because of Eq. 2 . The maximum entropy associated with $q$ possible events is thus:

$$
H_{\max }(X)=-\sum(1 / q) \log _{2}(1 / q)=\log _{2}(q) .
$$

These definitions are consistent with the information measure already proposed, namely, the length $k$ of the information message associated with an information considered as an equivalence class. The fundamental theorem of source coding, one of the most important results of information theory, bridges the two definitions. The source which generates messages is modelled in Shannon's information theory as a random variable $X_{\mathrm{s}}$ where the event $x_{i}$ is the choice of the $i$-th symbol in the alphabet of size $q$. Let $H\left(X_{\mathrm{s}}\right)$ denote the source entropy, in binary units (shannons) per symbol. Then, the fundamental theorem of source coding tells that a message of length $n$ can be reversibly transformed into a binary message of average length $\bar{\ell}=n H\left(X_{\mathrm{s}}\right) \log _{2}(q)$. If $n$ is very large, the actual length is with high probability close to its average, hence we may identify $\bar{\ell}$ with the length $k$ of the information message associated with the original sequence of length $n$, since no shorter sequence is equivalent to it.

The recourse to probabilities is necessary in communication engineering for two main reasons: first, the messages to be transmitted must be dealt with as random since the message that will be sent is not known in advance; second, the presence of noise entails that reception is a probabilistic operation. This does not mean however that the concept of information entirely depends

\footnotetext{
${ }^{3}$ Prior to information theory, an entity named entropy has been introduced in physics. We shall briefly examine the relationship between physical and informational entropies in the next section.
} 
on probabilities. When we introduced above an information as a class of equivalent sequences, no probabilities had to be assigned to sequences. As regards associating an information quantity with a given sequence, the algorithmic information theory measures it as the length, referred to as algorithmic complexity, of the shortest programme which instructs a universal computer to generate this sequence (Chaitin 2005). This definition has its roots in computer science where noise is generally ignored. It is of great theoretical interest because it does not rely on probabilities, but the algorithmic complexity is generally not computable, which limits its practical usefulness. Although the probabilistic definition of an information quantity and the algorithmic one are entirely different, it can be shown that they provide almost the same results in instances where both are relevant, at least when applied to long enough sequences.

\section{Informational versus Physical Entropy}

The source of Shannon's paradigm generates information by choosing one among $M$ objects, $M \geq 2$, so its outcome may be modelled as a random variable, the choice of the $m$-th object being its $m$-th realization, occurring with probability $p_{m}$. Then, the average quantity of information produced by the source is its entropy (see Eq. 3 with $M$ substituted for $q$ in the formula). If all symbols are chosen with the same probability $1 / M$, the source entropy, according to Eq. 4, is $H=\log _{2} M$ shannons.

Seventy years before Shannon founded information theory, Boltzmann expressed the entropy $S$ of a physical system possibly assuming one of $W$ distinct states (or 'complexions'), according to statistical physics, as proportional to the logarithm of the number of states:

$$
S=k \log W,
$$

where $k$ is a constant (this formula is engraved on Boltzmann's tomb in Vienna). With different notations, this formula is identical to Shannon's entropy of a source where the choices are equiprobable, namely, Eq. 4. It is this formal identity which reportedly prompted von Neumann to advise Shannon to name 'entropy' his statistical measure of information.

Boltzmann's entropy measures the uncertainty about the state of a macroscopic system in the absence of any means to actually know it. Shannon's entropy, on the contrary, measures the uncertainty prior to the source outcome which resolves it. Information thus appears as measuring resolved uncertainty, whereas Boltzmann's entropy measures basically unresolved uncertainty. This remark led Brillouin to rename Shannon's entropy 'negentropy' (negative entropy) as measuring how much uncertainty has been cancelled by an information-bearing event (Brillouin 1956). This suggests that information can possibly diminish the physical entropy by an amount equal to Shannon's entropy.

Many (including myself) are convinced by this argument, others think that the identity of Eqs. 4 and 5 is purely formal and does not express any reality. 
For instance, Yockey nicely mocks the opinion quoted above, writing: 'Life does not feed on negentropy as a cat laps up cream' (Yockey 2005, p. 32). We shall try in "The Operation of Life Decreases the Physical Entropy", below, to make more explicit the relationship of the physical and informational entropies, showing that life permanently struggles against physical entropy by informational means. Assuming moreover that life, and only life, produces information leads to the conclusion that life thrives at the expense of physical entropy.

\section{Information in the Biological Context}

We stated in "The Living versus Inanimate Divide" that information can be annihilated, but also that it can be copied, hence can proliferate. These $a$ priori properties of information, of abstract and general character, radically differentiate it from matter and energy. We also laid emphasis on the necessity that information be physically inscribed on some support.

Let us now consider the case, of biological interest, where an information message is associated with a 'recipe', understood as the sequence of instructions which need be obeyed in order to construct some object. Then a bit or a block of bits in this message instructs a step of the construction process, hence its semantic content entirely depends on its location in the message. Notice that a location is defined only in a unidimensional object like a sequence, so a recipe cannot be borne by an object having more than a single dimension. If the object a recipe enables fabricating has more than a single dimension, then it cannot itself act as a recipe. Insofar as it has been entirely specified by the recipe, however, it bears at least the same amount of information as the recipe itself. The information borne by a fabricated object will be referred to as structural, or Aristotelian, as opposed to the symbolic information borne by the sequence associated with the recipe.

In biology, DNA is an information support of capital importance. The symbols of its alphabet are molecules, namely, the four nucleic bases $\mathbf{A}$, T, G and C. DNA exhibits an outstanding ability to be faithfully copied. The basic copying mechanism consists of splitting the complementary strands of its double helix (which requires little energy because their coupling by hydrogen bonds is weak) and to reconstruct the complementary strand of each of them, used as a template. Extremely efficient 'proof-reading' mechanisms ${ }^{4}$ eventually result in an almost perfectly faithful copy.

DNA acts precisely as a recipe, since a gene instructs the construction of a protein, and the whole genome that of a phenotype. A gene or a genome thus bears symbolic information, whereas a protein or a phenotype bears structural information borrowed from the symbolic information of the gene or the genome. The properties of annihilation and proliferation are thus specific

\footnotetext{
${ }^{4}$ Not to be confused with genomic error-correction mechanisms which are needed for correcting errors of other origin than the replication process (Battail 2007, 2008a, c).
} 
to the DNA-borne symbolic information. The structural information can only be annihilated when its support, i.e., the fabricated object, is destroyed or damaged, not by merely substituting some new information for the old one; and, unlike symbolic information, it can neither be copied nor instruct the construction of an object identical to it. Von Neumann has indeed shown that replicating any object demands that it contains its own symbolic description (von Neumann 1966), hence the duality genome/phenotype is necessary in living beings (Pattee 2007) as well as in artificial self-reproducing systems (Mange et al. 2004).

We already introduced the concept of potential information to designate the information borne by any occurring event. Such an event provides usable information only when it results in the recording of some sequence of discrete symbols, i.e., in symbolic information in the above meaning. The following figure depicts the relationship of potential, symbolic and structural information. Neither potential nor structural information can be copied. The transformation of potential into symbolic information involves recording; the transformation of symbolic into structural information needs semantics (Fig. 2).

We already noticed that a specific feature of symbolic information as, e.g., borne by DNA, is that its support is unidimensional. Any symbol in an information message has thus a well defined place in it. This property is lost in higherdimensional spaces, and it turns out that it is needed for assigning semantics by means of the tree which represents the information message, as alluded to above. More generally, a relation of order is needed so as to express syntactic rules, which are themselves needed for any description or specification. A protein is a 3-dimensional object, and a phenotype may be thought of as having 4 dimensions, if we include time as a relevant dimension besides the spatial ones (in order to account for the object's development; then, the irreversibility of time is another reason for the inability of structural information to be copied). The impossibility of copying proteins can be thought of as the actual content of the 'central dogma of molecular genetics'. For the same reason, a similar impossibility exists at the phenotype level, which widely extends its reach.

Living beings are characterized by self-maintaining and self-constructing processes, transforming the symbolic information of their genome into the structural information of their phenotype. Paradoxically, this dynamic behaviour mainly results in maintaining mature phenotypes in their present state, just like Lewis Carroll's Red Queen must run to stay where she is. The process of increasing physical entropy tends permanently to dissolve structures, and life permanently struggles to counteract this process. Although it is reasonable

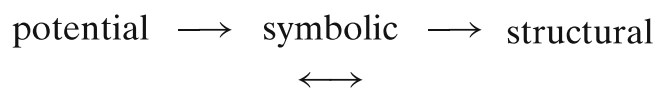

Fig. 2 Irreversibility of information transfer (or extended central dogma). Only symbolic information can be copied, as indicated by the double arrow. The arrow at left implies recording and crosses the border between the inanimate and the living worlds. The arrow at right implies semantics 
in our perspective to associate human-made machines with the living world, the extant ones still lack the property of self-maintenance that actual living beings possess. These machines only deal with an information coming from outside, but fail to process intrinsic information for lack of a reflexive function. Future machines will undoubtedly be endowed with self-maintenance ability.

\section{What Is Life?}

We dare entitling this section as Schrödinger's famous essay (Schrödinger 1943). The tremendous development of information technology and that, closely correlated, of information theory took indeed place after it has been published. The formal results of information theory, but also the wisdom that communication engineers have gained in practicing their art, considerably enriched the conceptual toolbox available for attacking the problem. We thus hope to be able to outline a plausible answer to this difficult question.

We distinguished above symbolic information which possibly bears the sequence of instructions needed for specifying a structure (a 'recipe') and can be copied; and structural information, which is borne by a structure thus specified and which, contrary to symbolic information, cannot be copied nor specify a structure.

Because information needs be physically inscribed on some support, physical structures are needed for implementing any operation involving information. Once we identify the living world (properly extended) as the exclusive place where information resides, such structures necessarily belong to it. They must be specified by symbolic information.

The genome contains this symbolic information, written on DNA molecules. It acts as a recipe for synthesizing the phenotypic structures which are needed for implementing:

- the conversion of the symbolic information borne by the genome into the phenotypic structures, using the proper semantic rules;

- the maintenance of these structures, using the same symbolic information and the same rules;

- the proliferation of the genome itself, by its regeneration based on the genomic error-correcting code (Battail 2007, 2008a, c) and its replication (including proof-reading).

Furthermore, the phenotype contains the DNA molecule which supports the genomic information and shields it from outer mechanical and chemical disturbances.

We may then depict life according to the scheme of Fig. 3.

The correspondence between the symbolic information of the genome and the phenotypic structures entirely depends on the location of symbols within the genome, which is unambiguously defined because its physical support, the DNA molecule, is a unidimensional object. This property is lost in objects of higher dimension, especially in the specified structures. Contrary 


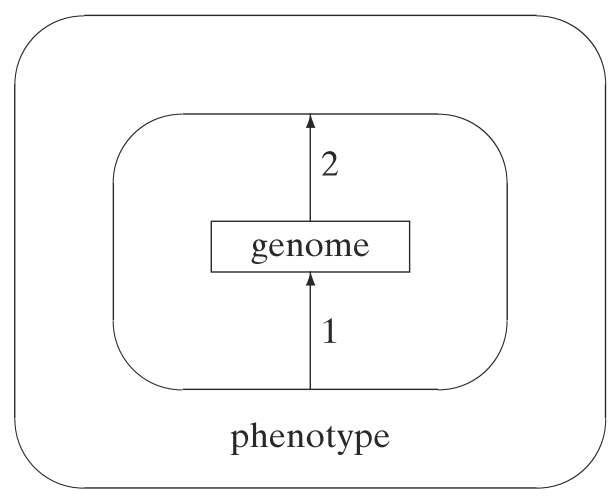

Fig. 3 Genome and phenotype: a schematic picture of life. The phenotype has been represented as a closed object which shields the genome it contains from the outside. The arrows depict their interaction: arrow 1 is intended to mean that the molecular machinery of the phenotype implements the genome regeneration and replication; arrow 2, that the symbolic information contained in the genome instructs the construction and maintenance of the phenotype

to information-bearing messages, the semantic rules need not be written. It suffices that a proper phenotypic machinery implements them. We may thus think, using a jargon borrowed from electrical engineering, that the semantic rules are 'wired' in the phenotypic machinery. As bearing the symbolic information which specifies this machinery, the genome implicitly contains the semantic rules.

\section{Consequences on Physics}

\section{The Operation of Life Decreases the Physical Entropy}

Physics assumes that the physical world has an objective existence, i.e., excludes solipsism, and uses the two main entities of matter and energy in order to describe it. The interaction of objects of the physical world are described by the laws of physics and chemistry. Both matter and energy are conserved, i.e., the quantity of matter (or energy) within some enclosure is constant. It changes only if matter (or energy) is introduced from outside, or taken out. A third physical entity, referred to as entropy, is more elusive. It measures how disordered is a set of objects. As an example, the distribution of the molecules of a gas within some closed volume is more or less disordered (see Fig. 4). The actual number of molecules is huge in any macroscopic system. For instance, one gramme of dihydrogen contains about $3 \times 10^{23}$ molecules. If the volume is initially divided into two compartments, one of which contains gas molecules whereas the other is empty, the kinetic theory of gases tells that eventually the gas molecules will evenly occupy the whole volume of both compartments when a hole is made in the separating wall. The initial state is then more ordered (its entropy is less) than the final one. The second law of 


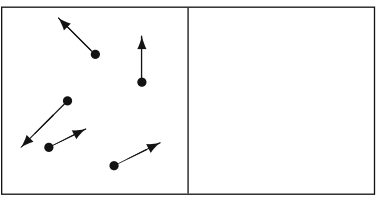

a

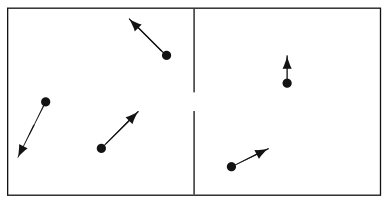

b

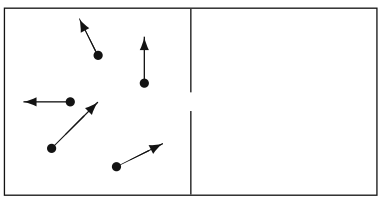

C

Fig. 4 Gas molecules within an enclosure (a molecule is represented as a black dot and its instantaneous speed vector is shown). a Initial state, two separated compartments, all gas molecules are at left. b A hole is made in the wall which separates the compartments. The molecules then tend to be evenly distributed within the whole volume; the physical entropy increases. c All molecules are located again in the left compartment. It is highly improbable, although not strictly impossible, that this situation spontaneously occurs: a thermodynamic miracle. Assumed to be able to individually control the molecules, Maxwell's demon could turn this exception into the rule, hence decrease the entropy

thermodynamics tells that this is a general behaviour: the entropy of an isolated system can but spontaneously increase. Such a system thus tends towards a state of maximal entropy, i.e., of maximal disorder. Notice that entropy is a mean quantity associated with a probabilistic system, hence its significance is statistical. In the above example, it may occur that at some instant all the gas molecules are located in one of the two previously separated compartments (see Fig. 4c). However, because of the huge number of molecules, this event would be a 'thermodynamic miracle' of extremely low probability, never observed in practice although possible in principle.

An isolated system is a mere fiction since, for instance, no shielding can protect from gravitational forces. This seems to severely restrict the cases to which the second law can rightfully be applied. However, besides its formal statement, the second law of thermodynamics can be loosely interpreted, and understood as stating that physical systems generally tend towards a state of increased entropy, i.e., tend to mixing and uniformity. It is clear from even the daily experience that the trend towards disorder is a universal reality of the inanimate world, even for physical systems which cannot be considered as isolated. This trend can be counteracted only by a deliberate control which needs information, especially at the atomic or molecular level. Only an information-receiving agent, dubbed Maxwell's demon, can thus exert it, for instance in the device of Fig. 4 by controlling a shutter so as to let molecules go from the right compartment to the left one through the hole, but not from left to right. A plentiful literature has been devoted to this fictitious object which violates the second law of thermodynamics (see Leff and Rex 2003). Most physicists deem that it cannot be implemented (e.g., Brillouin 1951). They paid little attention to the fact that life actually does so. Although the second law of thermodynamics is well verified as regards a wide class of physical systems, living beings constitute indeed an exception to it. Since living things are open systems which exchange matter and energy with their environment, they actually do not satisfy the conditions of validity of this law. Nevertheless, the process of life keeps them far from the state of maximal disorder during 
large intervals of time. Moreover, the living world tends to contain more and more numerous and differentiated objects, so that its whole physical entropy tends to decrease. The existence of life on Earth suffices to put and maintain it out of thermodynamic equilibrium, e.g., with an oxygen-rich atmosphere.

According to the reinterpretation of semiosis proposed in "A ReceiverOriented Interpretation of Semiosis", the basic tenet of biosemiotics implies that information-receiving objects exclusively belong to the living world, so its combination with the above statement that only an information-receiving agent can violate the second law of thermodynamics entails that

\section{living things, and only living things, can decrease the physical entropy.}

In other words, the living world is populated with Maxwell's demons. Although physicists imagined Maxwell's demon as external to the physical system on which it operates, however, a living thing is both this system and the demon, as noticed by Wiener (1948, p. 58).

We now illustrate how the very operation of life results in decreasing the physical entropy. As mere examples, we consider three instances: the synthesis of a protein, the self-reproduction of living beings, and the evolution of a population.

As a first and paradigmatic example, a clear illustration of the above statement is provided by the gene-instructed synthesis of a polypeptidic chain (eventually becoming a protein) which occurs in the cell. We assume that the 20 amino-acids which make up proteins are present, each in sufficient quantity. The number of distinct a priori possible polypeptidic chains of length $n_{\mathrm{p}}$ is $20^{n_{\mathrm{p}}}$, a huge number since a realistic order of magnitude of $n_{\mathrm{p}}$ is a few hundreds. The physical entropy associated with a mixture of $n_{\mathrm{p}}$ amino-acids is at most $S_{\mathrm{p}}=$ $\log _{2}\left(20^{n_{\mathrm{p}}}\right)=n_{\mathrm{p}} \log _{2}(20)$ (approximately $\left.n_{\mathrm{p}} \times 4.322\right)$ binary units. Now consider the translation process occurring within a cell. A molecule of messenger RNA (mRNA) uniquely determines a sequence of amino-acids which is assembled by the joint action of a transfer RNA (tRNA) molecule corresponding to each codon of the mRNA according to the genetic 'code', which binds itself to the amino-acid specified by this codon, and of the ribosomic machinery which binds together the amino-acids in the order of the mRNA codons which specify them. Then a unique polypeptidic chain is synthesized which replaces an initial mixture of $n_{\mathrm{p}}$ amino-acids, thus cancelling an amount of at most $S_{\mathrm{p}}=n_{\mathrm{p}} \log _{2}(20)$ binary units in the physical entropy of the system. The length $n_{\mathrm{p}}$ of each synthesized polypeptidic chain is actually determined by the position of a 'stop' codon in the mRNA molecule. Every time a polypeptidic chain of length $n_{\mathrm{p}}$ is synthetised, the entropy of the initial mixture of aminoacids decreases by at most $S_{\mathrm{p}}=n_{\mathrm{p}} \log _{2}(20)$. The cellular machinery results in decreasing the physical entropy because it controls individual amino-acid molecules. This may be thought of as a kind of Maxwell's demon. At variance with the system of Fig. 4 which contains identical molecules, however, the physical entropy results here from the mixing of different molecules and the ribosomic demon decreases the entropy by assembling a definite polypeptidic chain made of $n_{\mathrm{p}}$ of these molecules. 
The information quantity brought by the choice of one among $M$ objects, for instance with the same probability $1 / M$, is $H=\log _{2} M$. Letting $M=20^{n_{\mathrm{p}}}$ provides the informational entropy associated with the choice of a polypeptidic chain of length $n_{\mathrm{p}}$, namely, $H_{\mathrm{p}}=S_{\mathrm{p}}$, i.e., the translation of a gene by the cellular machinery provides a quantity of information which equals the physical entropy of the initial mixture of $n_{\mathrm{p}}$ amino-acids. Then the quantity of information has increased by the same amount as the physical entropy has decreased, which substantiates the equivalence of informational entropy with physical negentropy in this particular case (Brillouin 1956).

The example just given illustrates the relation of physical and informational entropies. It is simple enough to be quantitatively dealt with. The following examples are much more complicated, but they can be understood as instances of the paradigm provided by the first example. As a second example, the selfreproduction of living beings diminishes the physical entropy for just the same reason as the synthesis of a protein does. When a living thing bearing an amount of structural information of $H_{\text {ind }}$ is duplicated, the physical entropy decreases again by the same amount. As a result, the setting up of a population of $N$ identical individuals from successive replications of an ancester bearing an amount $H_{\text {ind }}$ of structural information results in a decrease in the physical entropy of $\mathrm{NH}_{\text {ind }}$. Notice that, although there is no more information in a set of $N$ identical objects than in each of them, the decrease in physical entropy equals the product of the information quantity that each object bears by the number of these objects.

A third example is again a population of $N$ individuals descending from a single ancestor, each bearing a quantity of structural information equal to $H_{\text {ind }}$, but we now assume that replication errors occur. A set of $N$ identical objects bears no more information than each of its elements, but the replication errors now result in more and more differing objects as their number $N$ increases, either with small differences frequently occurring (in the absence of a genomic error-correcting code), or with much more unfrequent but larger differences in the presence of such a code (Battail 2008a, c). Hence the existence of mutations in the population results in increasing the quantity of information contained in the population as a set of individuals when the size of this population itself increases. This increase of information quantity results from noise-generated errors, hence is again taken out from the physical entropy. The ability of individuals to convert symbolic into structural information results in an increase of the information quantity associated with the population diversity. Notice that the filtering operated by natural selection diminishes this quantity of information, in contradiction with Ronald Fisher's statement that 'Natural selection is a mechanism for generating an exceedingly high degree of improbability' (Fisher 1930).

The living world is organized as nested sets of objects. The molecular constituents of living tissues are mostly polymers made of small molecules, such polymers make up organelles, a cell is an assembly of organelles, an organ is a collection of cells, an individual is an assembly of organs, a population is a collection of individuals, etc, to name only a few. Besides the information 
borne by its individual constituents, each of these sets possesses its own information, borne by the differences between these constituents. Information thus exhibits the property of emergence. At any level, the information has been acquired at the expense of the physical entropy.

If the overall operation of life tends to decrease the physical entropy, however, implementing its functions needs energy hence involves metabolism, which increases to some extent the physical entropy, thus going against its trend to decrease.

\section{Other Consequences on Physics}

Carlo Rovelli's relational interpretation of quantum physics (Rovelli 2004) fully agrees with the picture of the physical world illustrated in Fig. 1. Any physical apparatus is a means for an observer located in the living world to receive information from the inanimate world. Such an apparatus is thus a channel in the information-theoretic meaning. Besides its own perspective, it has its own finite capacity which limits the rate at which it provides information hence defines its horizon.

According to the point of view defended in this paper, information is essential to properly account for the living world. Current physics fails to integrate information as a relevant fundamental entity, as it does for matter and energy, hence de facto excludes the living world from its domain of validity. The project of a 'theory of everything' is thus doomed to failure as a priori excluding a part of the physical world. If physics denies the living world, physicists deny themselves.

The culprit here is the implicit assumption that science's toolbox is complete, which sharply contradicts the openness that the progress of science demands: this is scientism, the intimate enemy of science. Assuming completeness is all the more dangerous since it remains implicit. It leads to scholarly aberrations which, alas, benefit from the authority of science.

\section{Conclusion}

Information appears as unavoidably needed for describing and understanding the living world, hence as central to biology. Physics ignores information as foreign to the inanimate world, and does not consider the living status of the observer as relevant. Paradoxically, mainstream biology is as blind as physics about the relevance of information, probably because physics acts as a parangon with respect to less formalized sciences. Although the word 'information' is ubiquitous in the biological literature, mainstream biology has not yet realized that information is a scientific entity indispensable for understanding life.

Indeed, the very existence of living things crucially depends on information, while the laws of physics and chemistry suffice to account for the interactions of the objects in the inanimate world. The living world, properly extended, 
appears as the sole place in the physical world where information can reside. It moreover acts as a trap for the physical entropy since all living beings behave as Maxwell's demons. Accepting information as a fundamental entity for describing the world is thus mandatory. For lack of doing so, physics has been able yet to describe and understand the inanimate world, leaving aside the living world. It attempts to integrate the observer as an actor of any measurement, especially at the quantum scale, but ignoring information results in denying the whole living world which includes the observer, hence contradicts the intended approach. On the other hand, considering information as a fundamental entity for describing the physical world enables integrating the living world into the vision of physics. Information then clearly delineates the border between the living and the inanimate worlds.

\section{References}

Barbieri, M. (2004). The definitions of information and meaning. Rivista di Biologia-Biology Forum, 97, 91-110.

Barbieri, M. (2008a). What is biosemiotics? Biosemiotics, 1(1), 1-3.

Barbieri, M. (2008b). The Scylla and Charybdis of biosemiotics. Biosemiotics, 1(3), 281-284.

Barbieri, M. (2008c). Biosemiotics: A new understanding of life. Naturwissenschaften, 95, 577-599.

Battail, G. (2007). Information theory and error-correcting codes in genetics and biological evolution. In M. Barbieri (Ed.), Introduction to biosemiotics (pp. 299-345). New York: Springer.

Battail, G. (2008a). Genomic error-correcting codes in the living world. Biosemiotics, 1, 221-238.

Battail, G. (2008b). Applying semiotics and information theory to biology: A critical comparison. Biosemiotics, 1(3).

Battail, G. (2008c). An outline of informational genetics. San Rafael: Morgan \& Claypool. http://www.morganclaypool.com/doi/abs/10.2200/S00151ED1V01Y200809BME023.

Boulding, K. E. (1956). The image. Ann Arbor: University of Michigan Press.

Brillouin, L. (1951). Maxwell's demon cannot operate: Information and entropy. I. In Leff and Rex, 2003 (pp. 120-123).

Brillouin, L. (1956). Science and information theory. London: Academic.

Chaitin, G. (2005). Metamath! New York: Pantheon.

Cover, T. M., \& Thomas, J. A. (1991). Elements of information theory. New York: Wiley.

Dawkins, R. (1982). The extended phenotype. Oxford: Oxford University Press.

Fisher, R. A. (1930). The general theory of natural selection. Oxford: Oxford University Press.

Leff, H. S., \& Rex, A. F. (Eds.) (2003). Maxwell's demon 2. Bristol: Institute of Physics Publishing.

Mange, D., Stauffer, A., Petraglio, E., \& Tempesti, G. G. (2004). Artificial cell division. Bio Systems, 76(1-3), 157-167.

Morange, M. (2007). Qu'est-ce que la vie extraterrestre? Sciences et Avenir, Hors-Série, 151, 36-40.

Pattee, H. H. (2007). The necessity of biosemiotics: Matter-symbol complementarity. In M. Barbieri (Ed.), Introduction to biosemiotics (pp. 115-132). New York: Springer.

Rovelli, C. (2004). Relational quantum theory. In N. Kolenda, \& A. Elitzur (Eds.), Quo vadis quantum mechanics? New York: Springer.

Schrödinger, E. (1943). In What is life? and Mind and matter. Cambridge; Cambridge University Press.

Shannon, C. E. (1948). A mathematical theory of communication. Bell System Technical Journal, 27, 379-457, 623-656.

von Neumann, J. (1966). In A. W. Burks (Ed.), Theory of self-reproducing automata. Urbana: University of Illinois Press.

Wiener, N. (1948). Cybernetics (2nd ed.). Cambridge: MIT and Wiley.

Yockey, H. P. (2005). Information theory, evolution, and the origin of life. Cambridge: Cambridge University Press. 